\title{
Type IV Effector Proteins Involved in the Medicago-Sinorhizobium Symbiosis
}

\author{
Matthew S. Nelson, ${ }^{1,2}$ Chan Lan Chun, ${ }^{1}$ and Michael J. Sadowsky ${ }^{1,3}$ \\ ${ }^{1}$ Biotechnology Institute; ${ }^{2}$ Department of Plant Biology; and ${ }^{3}$ Department of Soil Water \& Climate, University of Minnesota, \\ St. Paul, MN, U.S.A.
}

Accepted 28 November 2016.

In this study, we investigated genetic elements of the type IV secretion system (T4SS) found in Sinorhizobium spp. and the role they play in symbiosis. Sinorhizobium meliloti and $S$. medicae each contain a putative T4SS similar to that used by Agrobacterium tumefaciens during pathogenesis. The Cre reporter assay for translocation system was used to validate potential effector proteins. Both $S$. meliloti and $S$. medicae contained the effector protein TfeA, which was translocated into the host plant. Sequence analysis revealed the presence of a nod box involved in transcriptional activation of symbiosis-related genes, upstream of the transcriptional regulator (virG) in the Sinorhizobium T4SS. Replicate quantitative reverse transcription-polymerase chain reaction analyses indicated that luteolin, released by roots and seeds of Medicago truncatula, upregulated transcription of $t f e A$ and virG. Mutations in the T4SS apparatus or $t f e A$ alone resulted in reduced numbers of nodules formed on $M$. truncatula genotypes. In addition, $S$. meliloti KH46c, which contains a deletion in the T4SS, was less competitive for nodule formation when coinoculated with an equal number of cells of the wild-type strain. To our knowledge, TfeA is the first T4SS effector protein identified in Sinorhizobium spp. Our results indicate that Sinorhizobium i) uses a T4SS during initiation of symbiosis with Medicago spp., and ii) alters Medicago cells in planta during symbiosis. This study also offers additional bioinformatic evidence that several different rhizobial species may use the T4SS in symbiosis with other legumes.

Rhizobia form a well-studied symbiotic association with legume plants, resulting in the formation of nodules on roots or shoots that fix atmospheric nitrogen in exchange for plant-derived carbon. Sinorhizobium and Medicago spp. are often used as model organisms to study this symbiosis. Nodulation and nitrogen fixation requires the concerted efforts of both the plant and the bacterium, using genes involved in recognition, signal exchanges, nodulation, and nitrogen fixation (Saeki 2011). Plant-microbe interactions are complex and fluid and carry the potential to become commensal or pathogenic (Agrawal 2001). While rhizobia are often described as symbionts to legumes, they can be potential pathogens if nitrogen fixation levels drop below required thresholds.

Current address for C. L. Chun: Department of Civil Engineering, University of Minnesota-Duluth, Duluth, MN, U.S.A.

Corresponding author: M. J. Sadowsky; E-mail: sadowsky@umn.edu; Telephone: +1.612.624.2706.

*The $\boldsymbol{e}$-Xtra logo stands for "electronic extra" and indicates that one supplementary table is published online.

@ 2017 The American Phytopathological Society
Whole genome sequencing of symbiotic Rhizobium species strains have shown the presence of effector secretion systems, including types III, IV and VI (Nelson and Sadowsky 2015). Although several of these secretion systems are not essential for symbiosis, some have been shown to have both positive (increasing nodule number) and negative (reducing host range) effects on the symbiosis (Deakin and Broughton 2009).

Many bacterial species, including pathogens, use secretion systems to transport proteins into a eukaryotic host (Cascales and Christie 2003). Agrobacterium tumefaciens and A. rhizogenes use the type IV secretion system (T4SS) to transport effector proteins required for host infection (Berger and Christie 1994; Moriguchi et al. 2001). These proteins alter the host cell to improve virulence. Plants contain resistance $(R)$ genes that recognize these effector proteins and activate plant immune responses, which lead to pathogen resistance (Anderson and Frank 2012; Deslandes and Rivas 2012).

Bradyrhizobium elkanii contains the T3SS, which transports effector proteins during soybean symbiosis to increase total nodule number (Okazaki et al. 2009). Some soybean lines contain an $R$ gene that induces effector-triggered immunity, which prevents Bradyrhizobium elkanii with the T3SS from forming nodules (Yasuda et al. 2016). S. fredii HH103 was also shown to use the T3SS to suppress early defense response by soybean (Jiménez-Guerrero et al. 2015). These interactions are consistent with the plant pathogen zigzag model (Jones and Dangl 2006).

Mesorhizobium loti R7A contains a T4SS similar to that of Agrobacterium spp. and transports two effector proteins, Msi059 and Msi061, into the host cell (Hubber et al. 2004). Mutations in the Mesorhizobium loti T4SS or effectors resulted in a decrease in the number of nodules formed by Lotus corniculatus. However, Mesorhizobium loti T4SS mutants could form functioning nodules with Leucaena leucocephala, unlike the wild-type strain. These results suggest that L. leucocephala contains an $R$ gene for these effectors (Hubber et al. 2004).

Previous studies done on the T4SS in S. meliloti 1021 did not find any relationship between the T4SS and symbiotic effectiveness (Jones et al. 2007). However, Illumina sequencing of 48 Sinorhizobium strains revealed seven different subfamilies of the T4SS (Sugawara et al. 2013). The T4SSa functions in conjugation, while T4SSb is involved in effector protein transport. Since strain 1021 contained only T4SSa and not T4SSb, the lack of a role of T4SSb in symbiosis was, thus, expected. Other strains, however, such as S. meliloti KH46c and S. medicae M2, contain a T4SS that is phylogenetically similar to the T4SS found in Mesorhizobium loti R7A and Agrobacterium spp.

Only a few T4SS effectors have been identified and none has a known function in planta. Effector proteins identified in Sinorhizobium spp. are of particular interest because of the genetic modification tools available for its host, genus Medicago. 
An understanding of the role and function of effector proteins that are transported through the T4SS could provide new insight into how rhizobia communicate with and alter the function of plant cells to increase nodule formation. In this study, we report the identification of effector proteins in Sinorhizobium and the phenotypic effects that these proteins have on symbiosis with Medicago genotypes.

\section{RESULTS}

\section{Identification of potential type IV effector proteins.}

Effector proteins vary in function and composition between microorganisms, but a few aspects of effector proteins and the T4SS are conserved across species (Deakin and Broughton 2009). These conserved motifs and characteristics were used to identify potential effector proteins in the Sinorhizobium strains investigated in our studies. These characteristics included: i) genes for effector proteins are located near the T4SS genes, ii) effector proteins have a positively charged C-terminus (Vergunst et al. 2005), and iii) effector protein genes are found in most strains containing the T4SS but absent in strains lacking the T4SS. Using these criteria, five candidate genes were identified from $32 \mathrm{~S}$. meliloti strains and two candidate genes from $12 \mathrm{~S}$. medicae strains.

\section{Validation of type IV effector proteins.}

Previous researchers have demonstrated function of T4SS effector proteins in rhizobia by using the Cre Recombinase Assay for Translocation (CRAfT) system (Hubber et al. 2004). Genetic sequences that encoded for the 27-amino acid C-terminus of each candidate protein were fused to Cre (Supplementary Table $\mathrm{S} 1$ ). The Cre-containing plasmids were inserted into $A$. tumefaciens LBA 1100 by transformation and were cocultivated with Arabidopsis thaliana line 3043 (Vergunst et al. 2000). Results of three independent cocultivation experiments are shown in Table 1. Cocultivation with constructs that code for candidate 1 , from $S$. medicae, and candidate 4, from S. meliloti, produced 0.29 and 0.32 kanamycin-resistant calli per root explant, respectively. For comparison, cocultivation with the Cre:GALLS-27 positive control produced, on average, 0.36 kanamycin-resistant calli per root explant.

No kanamycin-resistant calli were recovered from cocultivation with constructs that code for any of the other candidate proteins. Similarly, cocultivation with Cre alone and calli from A. tumefaciens LBA2587 ( $\Delta$ virD4), did not produce any kanamycin-resistant calli (Beijersbergen et al. 1992; Vergunst et al. 2003). Taken together, these results show that the C-termini of candidate genes 1 and 4 contain the T4SS signal and translocate through the T4SS into the host cell.

\section{Distribution and synteny of T4SS genes among rhizobia.}

Candidate 1 and candidate 4 effector proteins shared $97 \%$ protein identity. These homologous proteins were named TfeA (type IV effector protein A). In the 48 strains examined by Sugawara et al. (2013), TfeA was found in all strains containing the T4SSb (in nine $S$. meliloti and $11 S$. medicae strains). The TfeA protein sequence is $100 \%$ conserved within the strains of each species. TfeA is located 1,669 and 1,533 bp upstream of virD4 in S. meliloti $\mathrm{KH} 46 \mathrm{c}$ and $S$. medicae $\mathrm{M} 2$, respectively. Both genes are 888 bp long (Fig. 1).

Nod boxes are conserved sequence motifs located upstream of genes involved in symbiosis and nodule formation (Goethals et al. 1992; Suominen et al. 1999). The nod box motif was found upstream of virG in S. meliloti KH46c and S. medicae M2 as well as in the other Sinorhizobium strains with the T4SS. This nod box was $84 \%$ identical (62 of $74 \mathrm{bp}$ ) to the promoter region between nodDl and nodA in $S$. meliloti and S. medicae. VirG in Agrobacterium spp. is the transcriptional activator responsible for inducing the T4SS apparatus and effector proteins by binding to vir boxes (Stachel and Zambryski 1986). There were no identified vir boxes upstream of any T4SS proteins, based on similarity to Agrobacterium vir boxes (Pazour and Das 1990).

\section{Upregulation of $\operatorname{vir} G$ and $t f e A$ by luteolin.}

The plant flavonoid luteolin is the first signaling molecule in the Sinorhizobium-Medicago nodule-formation signaling cascade (Peters et al. 1986). Sinorhizobium genes involved early in symbiosis are upregulated by exposure to luteolin (Barnett et al. 2004). To validate that the T4SS expression occurs early during symbiosis, quantitative reverse transcription-polymerase chain reaction (qRT-PCR) was performed after exposure to luteolin. Gene expression fold changes were determined at 0,4 , and $24 \mathrm{~h}$ postinduction (hpi) (Table 2). Since uppS expression levels were previously shown not to be affected by luteolin (Barnett et al. 2004), RNA expression levels were normalized to uppS. Results show that nodA, virG and $t f e A$ were induced about 2.5fold at $4 \mathrm{hpi}$, based on an average of two independent experiments (Table 2). After $24 \mathrm{hpi}$, there was an approximately sixfold increase in nodA and $t f e A$ gene expression. In contrast, $\operatorname{vir} G$ expression did not increase. These results show that $t f e A$ is induced by luteolin and further suggested that TfeA may play an early role in symbiosis.

\section{Phenotypic effects of targeted deletions in T4SS or candidate effector proteins.}

Targeted deletion mutants were used to determine the influence of effector proteins $(\mathrm{tfe} A)$ and theT4SS apparatus (B6-B9) on the symbiotic phenotypes of Sinorhizobium strains. Medicago genotypes A17 and R108 were inoculated with wildtype or mutant strains. Results show that, 6 weeks after inoculation, S. meliloti KH46c $\Delta B 6-B 9$ formed significantly fewer nodules relative to the wild-type strain on $M$. truncatula A17 $(P=0.006)$ but not on R108 $(P=0.057)$ (Table 3$)$. In contrast, strain KH46c $\Delta t f e A$ formed fewer nodules relative to the wild type on both A17 $(P=0.003)$ and R108 $(P=0.004)$. Likewise, $S$. medicae M2 $\Delta t f e A$ formed significantly fewer nodules $(P<$ $0.001)$ than the wild type on $M$. truncatula A17, whereas $S$. medicae M2 $\triangle B 6-B 9$ formed marginally fewer nodules than the wild-type strain on A17 $(P=0.056)$. Nodule weight (on a per-nodule basis) significantly increased in $S$. meliloti $\mathrm{KH} 46 \mathrm{c} \Delta B 6-B 9$ relative to the wild-type strain on $M$. truncatula R108 $(P=0.068)$ but not on A17 $(P=0.020)$, and strain

Table 1. The number of Arabidopsis thaliana 3043 root explants transformed to express kanamycin resistance after cocultivation with Agrobacterium tumefaciens containing different constructs

\begin{tabular}{lrl}
\hline Strain/plasmid & Transformed/total calli & \% Transformed \\
\hline LBA 1100 & $0 / 308$ & 0 \\
Cre & $210 / 583$ & 0.36 \\
Cre::GALLS & $72 / 251$ & 0.29 \\
Cre::Candidate $1^{\mathrm{b}}$ & $0 / 219$ & 0 \\
Cre::Candidate $2^{\mathrm{b}}$ & $0 / 208$ & 0 \\
Cre::Candidate $3^{\mathrm{c}}$ & $83 / 253$ & 0.32 \\
Cre::Candidate $4^{\mathrm{c}}$ & $0 / 185$ & 0 \\
Cre::Candidate $5^{\mathrm{c}}$ & $0 / 169$ & 0 \\
Cre::Candidate $6^{\mathrm{c}}$ & $0 / 176$ & 0 \\
Cre::Candidate $7^{\mathrm{c}}$ & & 0 \\
LBA 2587 & $0 / 323$ & 0 \\
Cre::GALLS & $0 / 214$ & 0 \\
Cre::Candidate $1^{\mathrm{b}}$ & $0 / 157$ & 0 \\
Cre::Candidate $2^{\mathrm{b}}$ & $0 / 124$ & 0 \\
Cre::Candidate $3^{\mathrm{c}}$ & $0 / 237$ & \\
Cre::Candidate $4^{\mathrm{c}}$ & & 0 \\
\hline
\end{tabular}

\footnotetext{
a The C-terminus was found in Agrobacterium rhizogenes

b The C-terminus was found in Sinorhizobium medicae.

c The C-terminus was found in Sinorhizobium meliloti.
} 
$\mathrm{KH} 46 \mathrm{c} \Delta t \mathrm{fe} A$ also formed larger nodules relative to the wild type on both genotypes A17 $(P<0.001)$ and R108 $(P=0.005)$. The $S$. medicae deletion mutant M2 $\Delta B 6-B 9(P=0.039)$ and M2 $\Delta t f e A(P=0.045)$ formed larger nodules than did the wildtype strain on $M$. truncatula A17.

Both mutant and wild-type strains produced pink nodules near the crown of plant roots, suggesting that the effector mutations did not delay the timing of nodulation or nitrogen fixation efficacy. The wild-type strains, however, continued to form nodules farther down the plant root system than did the mutants and these nodules were smaller and white (data not shown).

Dry mass of the Medicago plants inoculated with the mutant Sinorhizobium strains was similar to the plants inoculated with the wild-type strains $(P>0.05)$. None of the plants showed any type of visual stress response and chlorophyll content of Medicago plants inoculated with the mutant strains showed no significant difference $(P>0.05)$ from plants inoculated with the wild-type strains (Table 3 ).

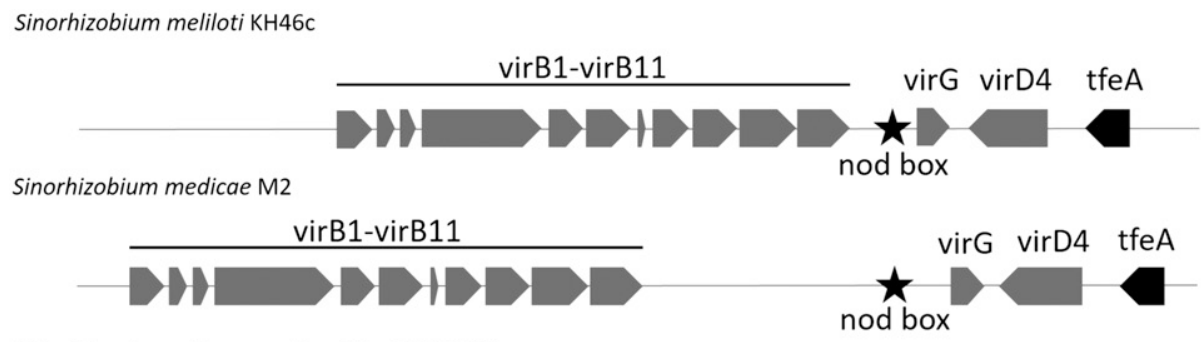

Rhizobium leguminosarum bv viciae TOM 3841

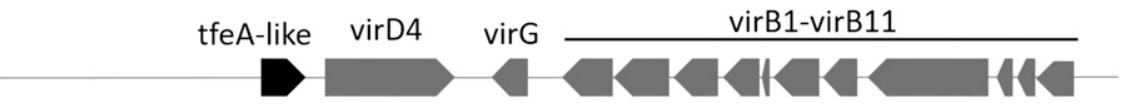

Mesorhizobium loti R7A

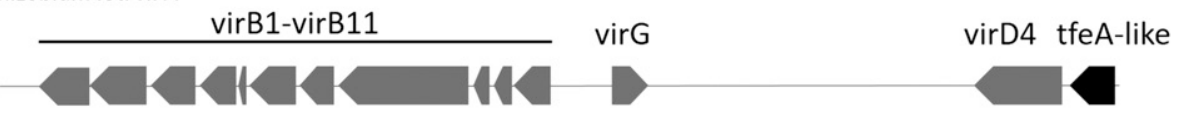

Mesorhizobium opportunistum WSM2075

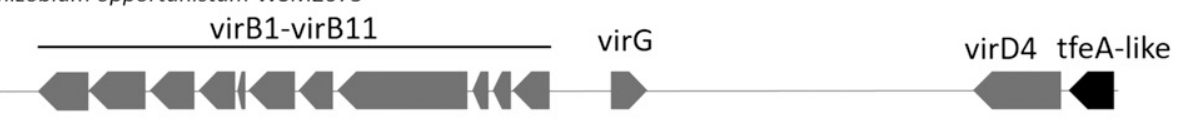

Mesorhizobium australicum WSM2073

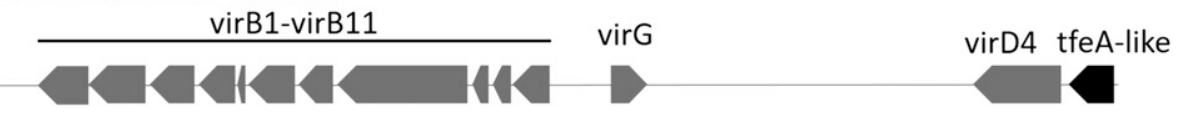

Mesorhizobium ciceri bv biserrulae WSM1271

virB1-virB11 virG virD4 tfeA-like

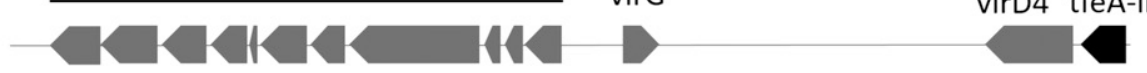

Rhizobium sullae WSM1592

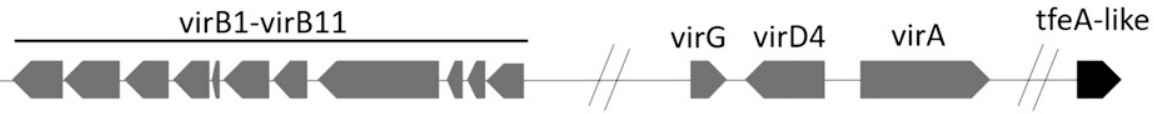

Rhizobium mesoamericanum STM3625

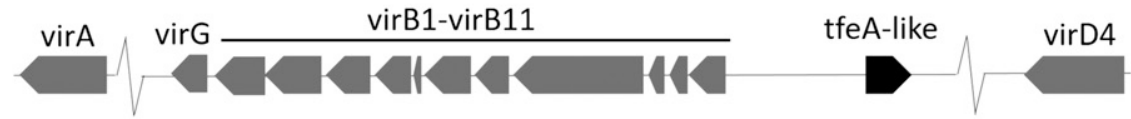

Sinorhizobium arboris LMG 14919

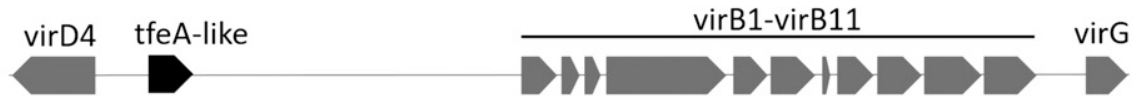

Rhizobium leguminosarum bv trifolii WSM2297

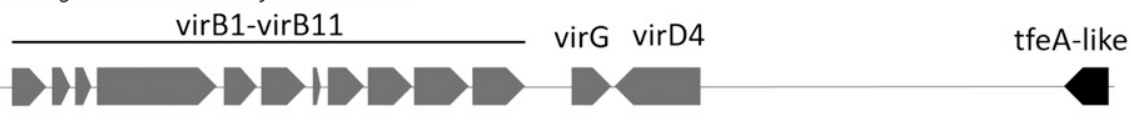

Rhizobium etli CFN 42 DSM 11541

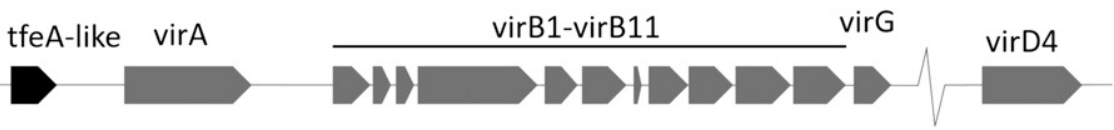

Fig. 1. Genetic organization of the type IV secretion system and surrounding genes in diverse rhizobial strains. The nod box promoter sequence is designated by asterisks (*) in both Sinorhizobium meliloti KH46c and S. medicae M2 strains. 
Competition for nodulation between the $S$. meliloti KH46c $\Delta B 6-B 9$ mutant and the wild-type strain.

While the T4SS is not found in all $S$. meliloti strains, its presence is associated with an increase in the total nodule number formed on 27 different Medicago genotypes (Sugawara et al. 2013). Coinoculation experiments were performed to determine if the T4SS offered a competitive advantage relative to strains that do not contain this secretion system. To aid in these studies, greenn or red fluorescent protein (GFP or RFP, respectively) were incorporated into the bacterial chromosome in a region known not to affect nodulation (Pistorio et al. 2002) and strains were inoculated onto Medicago genotypes A17 and R108. Wild-type KH46c (GFP) was coinoculated with an equal number of KH46c $\Delta B 6-B 9$ (RFP) cells and wild-type KH46c (RFP) was coinoculated with an equal number of strain KH46c $\Delta B 6-B 9$ (GFP) cells. Adequate fluorescence could not be detected from strains transformed with RFP; therefore, PCR was subsequently used for strain identification.

Table 4 shows nodule occupancy rates for each strain combination. Mutant strains KH46c $\triangle B 6-B 9$ labeled with GFP and RFP were isolated from $34 \%$ of the $M$. truncatula A17 nodules and $36 \%$ of the M. truncatula R108 nodules. In contrast, 66 and $64 \%$ of nodules were occupied by the wild-type strains on M. truncatula genotypes A17 and R108, respectively. Thus, the $\mathrm{KH} 46 \mathrm{c} \Delta B 6-B 9$ mutant strains were less competitive for nodulation than the wild-type strains, after 4 weeks of cocultivation.

\section{TfeA-like proteins found}

in other Rhizobium species strains.

The T4SS apparatus along with the virD4 gene that is required for protein translocation is found in multiple Rhizobium species (Nelson and Sadowsky 2015). A BLAST search was done to compare the TfeA amino acid sequence to those in

Table 2. Induction of Sinorhizobium meliloti nodD, virG, and tfeA genes by luteolin

\begin{tabular}{llll}
\hline & \multicolumn{3}{c}{ Fold gene induction in $\boldsymbol{S}$. meliloti $\mathbf{K H 4 6 \mathbf { c } ^ { \mathbf { a } }}$} \\
\cline { 2 - 4 } Hpi & $\boldsymbol{n o d \boldsymbol { A }}$ & \multicolumn{1}{c}{$\boldsymbol{\text { ir } \boldsymbol { G }}$} & $\boldsymbol{t} \boldsymbol{\text { e } \boldsymbol { A }}$ \\
\hline 0 & 0.71 & 0.62 & 0.76 \\
4 & $2.45 \pm 0.50$ & $2.5 \pm 0.02$ & $2.41 \pm 0.05$ \\
24 & $5.79 \pm 1.41$ & $1.09 \pm 0.31$ & $5.99 \pm 0.28$ \\
\hline
\end{tabular}

${ }^{\mathrm{a}}$ Expression is normalized to $u p p S$. Hpi $=$ hours postinduction. the Integrated Microbial Genomes, National Center for Biotechnology Information, and Microscope databases (Table 5). Protein sequences with 22 to $97 \%$ identity to TfeA across the entire length of the protein were found in nine different rhizobial species strains, representing four genera (Table 5). All of these species also contained the T4SSb effector-transporting subfamily. Table 5 also shows the percent identity of VirD4, as it varies less between the T4SS subfamilies. This is consistent with T4SS proteins sharing homology between species but effector proteins having very little homology between species. Homologs of VirD4 had 80 to $99 \%$ amino acid sequence identity. The TfeA-like proteins from the databases contained the T4SS secretion signal, which is required for protein translocation (Vergunst et al. 2005). This conserved region consisted primarily of positively charged arginine residues. Additionally, the $t f e A$-like genes were located in close proximity to virG and the T4SS apparatus (Fig. 1).

\section{DISCUSSION}

Determining the molecular interactions that occur between macro- and microsymbionts is important to better understand the totality of the symbiotic relationship. Here, we identified a new effector protein, TfeA, that is involved in the symbiosis of Sinorhizobium spp. with Medicago spp. We show that TfeA is transported through the T4SS into the plant cell and that expression of the $t f e A$ gene is upregulated in response to luteolin. This suggests that TfeA is involved in the early stages of symbiosis. Moreover, deletion of this effector reduced the number of nodules formed on $M$. truncatula, further showing its role in symbiosis. Despite the large effect that TfeA had on symbiosis, it was not found in all Sinorhizobium strains. We propose that the TfeA may be selected against by specific plant genotypes, as it has an effect on competitive interactions.

Table 4. Percent nodule occupancy by Sinorhizobium KH46c $\Delta B 6-B 9$ and the wild-type strain on two Medicago genotypes

\begin{tabular}{lcccc}
\hline & \multicolumn{3}{c}{ \% Nodule occupancy by strain } & \\
\cline { 2 - 4 } Host plant & KH46c & KH46c $\boldsymbol{\Delta} \boldsymbol{B 6}$ - $\boldsymbol{B}^{\mathbf{9}}$ & Both & Nodules tested \\
\hline A17 & 64.7 & $33.3^{* *}$ & 2 & 150 \\
R108 & 60.2 & $35.4^{* *}$ & 4.4 & 113 \\
\hline
\end{tabular}

a Asterisks (**) indicate $P<0.001 P$ values calculated using $t$ test (unequal variance)

Table 3. Symbiotic phenotype of Sinorhizobium mutant and wild-type (WT) strains ${ }^{a}$

\begin{tabular}{|c|c|c|c|}
\hline Phenotype & WT & $\Delta B 6-B 9$ & $\Delta t f e A$ \\
\hline \multicolumn{4}{|c|}{ Medicago truncatula A17 inoculated with Sinorhizobium meliloti KH46c } \\
\hline Nodule number & $34.25(2.80)$ & $22.5(2.38) *$ & $22.25(1.58) *$ \\
\hline Plant dry mass (grams) & $0.131(0.02)$ & $0.128(0.02)$ & $0.159(0.01)$ \\
\hline Chorophyll content (SPAD) & $43.9(0.89)$ & $43.5(0.86)$ & $44.1(0.89)$ \\
\hline Per nodule dry mass (milligrams) & $0.141(0.01)$ & $0.211(0.03)$ & $0.222(0.02)^{* *}$ \\
\hline Total plant nitrogen $(\%)$ & 4.106 & 4.012 & 4.08 \\
\hline \multicolumn{4}{|c|}{ Medicago truncatula $\mathrm{R} 108$ inoculated with Sinorhizobium meliloti KH46c } \\
\hline Nodule number & $28.88(2.88)$ & $22.38(0.60)$ & $17(1.73)^{*}$ \\
\hline Plant dry mass (grams) & $0.170(0.02)$ & $0.164(0.01)$ & $0.180(0.02)$ \\
\hline Chorophyll content (SPAD) & $36.4(0.54)$ & $35.9(0.65)$ & $34.6(0.74)$ \\
\hline Per nodule dry mass (milligrams) & $0.233(0.02)$ & $0.323(0.03) *$ & $0.355(0.03) *$ \\
\hline Total plant nitrogen $(\%)$ & 4.467 & 4.47 & 4.479 \\
\hline \multicolumn{4}{|c|}{ Medicago truncatula A17 inoculated with Sinorhizobium medicae M2 } \\
\hline Nodule number & $27.13(1.91)$ & $22.38(1.18)$ & $14.63(1.74)^{* *}$ \\
\hline Plant dry mass (grams) & $0.113(0.01)$ & $0.137(0.01)$ & $0.0887(0.01)$ \\
\hline Chorophyll content (SPAD) & $42(1.30)$ & $41.6(0.67)$ & $41.6(1.21)$ \\
\hline Per nodule dry mass (milligrams) & $0.139(0.01)$ & $0.192(0.02)^{*}$ & $0.202(0.02)^{*}$ \\
\hline Total plant nitrogen $(\%)$ & 3.824 & 3.972 & 4.139 \\
\hline
\end{tabular}

${ }^{a}$ One asterisk $(*)$ indicates $P<0.05$, two $(* *)$ indicate $P<0.001 . P$ values calculated using $t$ test (unequal variance). Standard error values are in parentheses. 
The T4SS in Mesorhizobium loti R7A is known to restrict host range (Hubber et al. 2004). The T3SS and T4SS effector proteins have been shown to repress or enhance nodule formation, depending on the host (Deakin and Broughton 2009). The T3SS in $S$. fredii USDA257 is recognized by the Glycine max rj2 gene, which prevents nodulation (Yang et al. 2010). It has also been suggested that, although these effector proteins are involved in the symbiotic process, they may also be used in pathogen-related responses (Marie et al. 2001; Nelson and Sadowsky 2015). If TfeA also acts in pathogenic relationships, its effects on altering the host cell may offer important insights into how Sinorhizobium spp. modify innate host immunity responses, allowing for nodule formation by perceived friends rather than foes.

Interestingly, TfeA has $25 \%$ amino acid identity to $\mathrm{VirF}$ in A. tumefaciens C58. This A. tumefaciens protein is involved in targeted proteolysis by binding to VIP1, an A. tumefaciens effector, and Skp1p, a subunit of E3 ubiquitin ligase complex (Schrammeijer et al. 2001; Tzfira et al. 2004). Effector proteins could potentially target plant proteins to alter host cells in a manner beneficial to the bacteria.

BLAST analysis showed that nine different rhizobial species contained a TfeA-like protein. These proteins were always found associated with the T4SS and were often found in close genetic proximity to the T4SS (Fig. 1). Although not all the TfeA-like proteins have been experimentally proven to translocate into plant cells, they all contain the signal motif for translocation (Vergunst et al. 2005). In Mesorhizobium loti R7A, protein Msi061 has been shown to translocate and has 59\% amino acid homology to TfeA (Table 5). Msi061 also has a similar effect on symbiosis as did TfeA. Together, this suggests that a wide range of rhizobia species are modifying different hosts in a similar manner.

We show here that deleting $t f e A$, which encodes for candidate proteins 1 and 4 , significantly reduced the total number of nodules formed on Medicago spp. The nodules that did form were significantly larger than those formed by the wild-type strain, suggesting that the plant increased the amount of carbon provided to each nodule in a compensatory manner (Kiers et al. 2003; Singleton and Stockinger 1983).

While TfeA does not appear to influence plant fitness, the T4SS affected competitiveness for nodulation. The wild-type $S$. meliloti KH46c strain was more competitive at forming nodules than the $\triangle B 6-B 9$ deletion mutant. Thus, while possession of a T4SS may appear to confer a selective advantage to Sinorhizobium strains, the secretion system is not found in all Sinorhizobium strains and its distribution does not cluster on the phylogenetic tree (Sugawara et al. 2013). Consequently, the T4SS may be a recent addition to Sinorhizobium or the T4SS may have been gained and lost at various points in the evolution of this bacterium. Along these lines, studies done concerning
T3SS in rhizobia suggest that the T4SS could be negatively selected for by a host and subsequently lost from some Sinorhizobium strains (Nelson and Sadowsky 2015).

Luteolin, an early symbiosis signaling molecule, acts on genes containing the nod-box promoter sequence. A nod-boxlike sequence was found upstream of virG in S. meliloti and $S$. medicae, which suggests that T4SS is likely upregulated early in symbiosis. Previous studies found that expression of the T4SS in Mesorhizobium loti R7A was regulated by a nodbox found 851 bp upstream of virA (Hubber et al. 2007). Similarly, in a phylogenetically unrelated strain, we found that tfeA and virG were both upregulated by exposure to luteolin. However, virG expression was only transiently upregulated $4 \mathrm{hpi}$ and after 24 hpi. These results are consistent with those found in Mesorhizobium loti R7A, in which virG expression was not significantly increased (Hubber et al. 2007).

This research clarifies the role of the T4SS in the genus Sinorhizobium, a model symbiotic bacterium. Effector proteins are increasingly being shown to have a large impact on symbiosis, particularly nodule number. Understanding the molecular action of effector proteins may provide further insights into host-microbe signal exchange and fine control on symbiotic processes.

\section{MATERIALS AND METHODS}

Identification of candidate proteins for the CRAfT assay.

Candidate proteins that might be translocated through the T4SS system were identified using Sinorhizobium sequence data in MaGe (Magnifying Genome). The following criteria were used to identify DNA sequences that code for candidate proteins: i) sequences are located within $20 \mathrm{~kb}$ of T4SS, ii) sequences are present in multiple strains of the same species, iii) strains with candidate sequences have a T4SS, and iv) sequences code for a positively charged $\mathrm{C}$-terminus with at least two arginine residues (Vergunst et al. 2005). Based on these criteria, we identified five and two candidate effector proteins from $S$. meliloti and S. medicae, respectively.

\section{Construction of plasmids for CRAfT assay.}

Plasmids containing the nucleotide sequence for the 27amino acid C-terminus from each candidate gene were constructed as described by Hodges et al. (2006). The sense and antisense DNA sequence for each C-terminus was purchased from Integrated DNA Technologies (Coralville, IA, U.S.A.). Oligos were designed to contain TCGA on the $5^{\prime}$ end of the sense strand and CTAG on the $3^{\prime}$ end of the antisense strand. Strands were annealed so that the nucleotide overhangs matched the overhangs created by restriction enzymes SalI and XbaI. The double-stranded DNAs were ligated into SalI/XbaI-digested pSDM3197

Table 5. TfeA-like proteins with the required conserved C-terminus amino acid motif present in multiple rhizobial species

\begin{tabular}{|c|c|c|c|}
\hline Species & TfeA $(\%)$ & VirD4 (\%) & 27-Amino acid C-terminus ${ }^{a}$ \\
\hline Sinorhizobium meliloti $\mathrm{KH}_{46 \mathrm{c}^{\mathrm{b}}}$ & & & -ISHAYNHAREDLIASSRSRDRADGTGR- \\
\hline S. medicae $\mathrm{M} 2^{\mathrm{b}}$ & 97 & 99 & -ISHAYNHAREDLIASSRSRDRADGSGR- \\
\hline Rhizobium leguminosarum bv. viciae TOM & 70 & 85 & -AYNHARDDLRPSSRSRDAAGSRDRTGR \\
\hline Mesorhizobium loti $\mathrm{R} \mathrm{A}^{\mathrm{b}}$ & 59 & 81 & GRDISHSYNHAREDLMEARRSRDRTGR- \\
\hline Mesorhizobium opportunistum WSM2075 & 55 & 81 & GQAINRSYSQARADLQESTRNRDRGGR_- \\
\hline Mesorhizobium australicum WSM2073 & 55 & 81 & GQAINRSYSQARADLQESTRNRDRGGR_- \\
\hline Mesorhizobium ciceri bv. biserrulae WSM1271 & 55 & 81 & GQAINRSYSQARADLQESTRNRDRGGR- \\
\hline Rhizobium sullae WSM1592 & 42 & 90 & -SISQVSDQARTDLVASFRSRERSDAGR- \\
\hline Rhizobium mesoamericanum STM3625 & 30 & 80 & -ESLNRADSDARSELASSSRSRER-NWGR- \\
\hline Sinorhizobium arboris LMG 14919 & 29 & 85 & -RLNRADNDARADLASSSRSRERSNWGR—- \\
\hline Rhizobium leguminosarum bv. trifolii WSM2297 & 25 & 84 & -EHLNRAENEARADLAS-FRSRERSDRGR_- \\
\hline Rhizobium etli CFN 42, DSM 11541 & 22 & 82 & -ATTEAGEMSRAELMSATRPRRQYDEGR - \\
\hline
\end{tabular}

\footnotetext{
a Amino acid sequencing aligned using Clustal Omega.

${ }^{\mathrm{b}}$ Proteins shown to translocate through the type IV secretion system.
} 
(Schrammeijer et al. 2003), and the resulting plasmid constructs were verified by sequencing at the University of Minnesota Genomics Center (St. Paul, MN, U.S.A.).

\section{Protein translocation using the CRAfT.}

Plasmid pSDM3197, Agrobacterium strains, and Arabidopsis seeds were provided by P. Hooykaas and W. Ream (Hodges et al. 2006; Vergunst et al. 2000). Plasmids were inserted into A. tumefaciens strains LBA1100 and LBA 2587, using electroporation as previously described (Hubber et al. 2004). Cocultivation and Cre recombinase-mediated restoration of kanamycin resistance were performed as previously described (Vergunst et al. 2000).

\section{Construction of candidate gene knockouts in Sinorhizobium spp.}

Targeted deletions of Sinorhizobium genes were obtained by homologous recombination as previously described (Prentki and Krisch 1984; Sugawara et al. 2013). The 600- to 800-bp regions flanking the genes to be deleted were amplified by PCR. The region upstream of each gene was digested using $E c o$ RI and BamHI. The region downstream of each gene was digested using BamHI and HindIII. The resulting fragment was inserted into the EcoRI/HindIII sites of plasmid pk18mob, was digested with BamHI, and was ligated with the BamHI fragment from the $\Omega$ interposon containing a Spec/Strep cassette. The mutants $S$. medicae M2 $\Delta t f e A$ and $S$. meliloti KH46c $\Delta t f e A$ were created by using homologous recombination that replaced the target gene with a Spec/Strep cassette (Prentki and Krisch 1984). Recombinant strains were selected on tryptone yeast (TY) agar plates containing $20 \mu \mathrm{g}$ chloramphenicol, $100 \mu \mathrm{g}$ spectinomycin, and $100 \mu \mathrm{g}$ streptomycin per milliliter.

\section{Nodulation phenotypes of Sinorhizobium strains with Medicago spp.}

Seeds of Medicago truncatula genotypes A17 and R108 were prepared as previously described (Bucciarelli et al. 2006). Plant assays were done in sterile Leonard jars containing a 1:1 mixture of Sunshine \#5 (SunGro Horticulture Inc., Vancouver, Canada) and Turface MVP (Profile Product LLC, Buffalo Grove, IL, U.S.A.). Plants were inoculated as previously described (Sugawara and Sadowsky 2014) and were grown for 6 weeks in a growth chamber. Total plant nitrogen was determined by the Research Analytical Laboratory of the University of Minnesota (St. Paul, MN, U.S.A.).

\section{Construction of GFP- or RFP-tagged Sinorhizobium spp.}

The DNA sequence containing RFP was amplified from vector pPCRRFP6 by PCR, was digested with EcoR1, and was ligated into EcoRI-digested plasmid pMP6 (Palani 2011; Pistorio et al. 2002). The resulting plasmid, pmp7, was confirmed by sequence analysis. Plasmids pMP6 and pMP7 were transferred to Escherichia coli WSM3064 by heat shock. The transformed E. coli strains were cocultivated with Sinorhizobium spp. on TY agar plates. Transconjugants were selected on TY plates containing $9 \mu \mathrm{g}$ of tetracycline per milliliter and lacking diaminopimelic acid. Both GFP or RFP were incorporated into the chromosome of Sinorhizobium strains between the recA and alaS genes and were confirmed by PCR (Pistorio et al. 2002).

\section{Competition of nodulation assay.}

Medicago truncatula genotypes A17 and R108 were grown as described above. Sinorhizobium strains KH46c and KH46c $\triangle B 6-B 9$ were grown in TY medium to an optical density at $600 \mathrm{~nm}$ $\left(\mathrm{OD}_{600}\right)=0.7$. Strains were combined at a ratio of $1: 1$ and were inoculated onto surface-sterilized Medicago seeds to a final concentration of approximately $3 \times 10^{8}$ cells per seed. After 4 weeks of plant growth, nodules were harvested, were sterilized by immersion in sodium hypochlorite, and were streaked onto TY plates as described by Sugawara and Sadowsky (2014). Fluorescent microscopy and multiplexed colony PCR were used to examine nodule isolates for the presence of either or both GFP and RFP. Primers were designed using ApE. PCR products were visualized on $1 \%$ agarose gels.

\section{qRT-PCR assay.}

Sinorhizobium KH46c was cultured in Vincent's minimal medium at $28^{\circ} \mathrm{C}$. Overnight culture was diluted to $\mathrm{OD}_{600}=0.1$. Cultures were grown for $24 \mathrm{~h}$. Luteolin was added 0,4 , or $24 \mathrm{~h}$ prior to RNA collection, which was done using Qiagen RNeasy kits (Qiagen, Chatsworth, CA, U.S.A.). All RNA levels were normalized prior to conversion to cDNA. SuperScript II reverse transcription was used to generate cDNA (ThermoFischer Scientific, Waltham, MA, U.S.A.). qRT-PCR was performed using the iTaq Universal SYBR green supermix (BioRad, Hercules, CA, U.S.A.). Samples were run in triplicate on a StepOnePlus real-time PCR system and the products were confirmed using a melting curve analysis (Applied Biosystems, Grand Island, NY, U.S.A.).

\section{ACKNOWLEDGMENTS}

This study was funded, in part, by grant DBI-1237993 from The National Science Foundation. We thank P. Hooykaas for providing Arabidopsis thaliana 3043, pSDM3197, and Agrobacterium tumefaciens strains LBA1100 and LBA2587. We thank W. Ream for providing plasmid pDM7, A. Lagares for providing plasmid pmp6, and N. Palani for providing the RFP construct. We especially thank M. Taylor for critically reading this manuscript.

\section{LITERATURE CITED}

Agrawal, A. A. 2001. Phenotypic plasticity in the interactions and evolution of species. Science 294:321-326.

Anderson, D. M., and Frank, D. W. 2012. Five mechanisms of manipulation by bacterial effectors: A ubiquitous theme. PLoS Pathog. 8:e1002823.

Barnett, M. J., Toman, C. J., Fisher, R. F., and Long, S. R. 2004. A dualgenome symbiosis chip for coordinate study of signal exchange and development in a prokaryote-host interaction. Proc. Natl. Acad. Sci. U.S. A. 101:16636-16641.

Beijersbergen, A., Dulk-Ras, A. D., Schilperoort, R. A., and Hooykaas, P. J. J. 1992. Conjugative transfer by the virulence system of Agrobacterium tumefaciens. Science 256:1324-1327.

Berger, B. R., and Christie, P. J. 1994. Genetic complementation analysis of the Agrobacterium tumefaciens virB operon: virB2 through virB11 are essential virulence genes. J. Bacteriol. 176:3646-3660.

Bucciarelli, B., Hanan, J., Palmquist, D., and Vance, C. P. 2006. A standardized method for analysis of Medicago truncatula phenotypic development. Plant Physiol. 142:207-219.

Cascales, E., and Christie, P. J. 2003. The versatile bacterial type IV secretion systems. Nat. Rev. Microbiol. 1:137-149.

Deakin, W. J., and Broughton, W. J. 2009. Symbiotic use of pathogenic strategies: Rhizobial protein secretion systems. Nat. Rev. Microbiol. 7:312-320.

Deslandes, L., and Rivas, S. 2012. Catch me if you can: Bacterial effectors and plant targets. Trends Plant Sci. 17:644-655.

Goethals, K., Van Montagu, M., and Holsters, M. 1992. Conserved motifs in a divergent nod box of Azorhizobium caulinodans ORS571 reveal a common structure in promoters regulated by LysR-type proteins. Proc. Natl. Acad. Sci. U.S.A. 89:1646-1650.

Hodges, L. D., Vergunst, A. C., Neal-McKinney, J., den Dulk-Ras, A., Moyer, D. M., Hooykaas, P. J. J., and Ream, W. 2006. Agrobacterium rhizogenes GALLS protein contains domains for ATP binding, nuclear localization, and type IV secretion. J. Bacteriol. 188:8222-8230.

Hubber, A., Vergunst, A. C., Sullivan, J. T., Hooykaas, P. J. J., and Ronson, C. W. 2004. Symbiotic phenotypes and translocated effector proteins of the Mesorhizobium loti strain R7A VirB/D4 type IV secretion system. Mol. Microbiol. 54:561-574.

Hubber, A. M., Sullivan, J. T., and Ronson, C. W. 2007. Symbiosis-induced cascade regulation of the Mesorhizobium loti R7A VirB/D4 type IV secretion system. Mol. Plant-Microbe Interact 20:255-261.

Jiménez-Guerrero, I., Pérez-Montaño, F., Monreal, J. A., Preston, G. M., Fones, H., Vioque, B., Ollero, F. J., and López-Baena, F. J. 2015. The Sinorhizobium (Ensifer) fredii $\mathrm{HH} 103$ type 3 secretion system 
suppresses early defense responses to effectively nodulate soybean. Mol. Plant-Microbe Interact 28:790-799.

Jones, J. D. G., and Dangl, J. L. 2006. The plant immune system. Nature 444:323-329.

Jones, K. M., Lloret, J., Daniele, J. R., and Walker, G. C. 2007. The type IV secretion system of Sinorhizobium meliloti strain 1021 is required for conjugation but not for intracellular symbiosis. J. Bacteriol. 189: 2133-2138.

Kiers, E. T., Rousseau, R. A., West, S. A., and Denison, R. F. 2003. Host sanctions and the legume-rhizobium mutualism. Nature 425:78-81.

Marie, C., Broughton, W. J., and Deakin, W. J. 2001. Rhizobium type III secretion systems: Legume charmers or alarmers? Curr. Opin. Plant Biol. 4:336-342.

Moriguchi, K., Maeda, Y., Satou, M., Hardayani, N. S., Kataoka, M., Tanaka, N., and Yoshida, K. 2001. The complete nucleotide sequence of a plant root-inducing (Ri) plasmid indicates its chimeric structure and evolutionary relationship between tumor-inducing (Ti) and symbiotic (Sym) plasmids in Rhizobiaceae. J. Mol. Biol. 307:771-784.

Nelson, M. S., and Sadowsky, M. J. 2015. Secretion systems and signal exchange between nitrogen-fixing rhizobia and legumes. Front. Plant Sci. 6:491.

Okazaki, S., Zehner, S., Hempel, J., Lang, K., and Göttfert, M. 2009. Genetic organization and functional analysis of the type III secretion system of Bradyrhizobium elkanii. FEMS Microbiol. Lett. 295:88-95.

Palani, N. P. 2011. Metabolic regulation and genetic tools for bacterial neutral lipid production.

Pazour, G. J., and Das, A. 1990. Characterization of the VirG binding site of Agrobacterium tumefaciens. Nucleic Acids Res. 18:6909-6913.

Peters, N. K., Frost, J. W., and Long, S. R. 1986. A plant flavone, luteolin, induces expression of Rhizobium meliloti nodulation genes. Science 233: 977-980.

Pistorio, M., Balagué, L. J., Del Papa, M. F., Pich-Otero, A., Lodeiro, A., Hozbor, D. F., and Lagares, A. 2002. Construction of a Sinorhizobium meliloti strain carrying a stable and non-transmissible chromosomal single copy of the green fluorescent protein GFP-P64L/S65T. FEMS Microbiol. Lett. 214:165-170.

Prentki, P., and Krisch, H. M. 1984. In vitro insertional mutagenesis with a selectable DNA fragment. Gene 29:303-313.

Saeki, K. 2011. Rhizobial measures to evade host defense strategies and endogenous threats to persistent symbiotic nitrogen fixation: A focus on two legume-Rhizobium model systems. Cell. Mol. Life Sci. 68: 1327-1339.

Schrammeijer, B., den Dulk-Ras, A., Vergunst, A. C., Jurado Jácome, E., and Hooykaas, P. J. 2003. Analysis of Vir protein translocation from Agrobacterium tumefaciens using Saccharomyces cerevisiae as a model: Evidence for transport of a novel effector protein VirE3. Nucleic Acids Res. 31:860-868.

Schrammeijer, B., Risseeuw, E., Pansegrau, W., Regensburg-Tuïnk, T. J., Crosby, W. L., and Hooykaas, P. J. 2001. Interaction of the virulence protein VirF of Agrobacterium tumefaciens with plant homologs of the yeast Skp1 protein. Curr. Biol. 11:258-262.

Singleton, P. W., and Stockinger, K. R. 1983. Compensation against ineffective nodulation in soybean. Crop Sci. 23:69.

Stachel, S. E., and Zambryski, P. C. 1986. virA and virG control the plantinduced activation of the T-DNA transfer process of $A$. tumefaciens. Cell 46:325-333.

Sugawara, M., Epstein, B., Badgley, B. D., Unno, T., Xu, L., Reese, J., Gyaneshwar, P., Denny, R., Mudge, J., Bharti, A. K., Farmer, A. D., May, G. D., Woodward, J. E., Médigue, C., Vallenet, D., Lajus, A., Rouy, Z., Martinez-Vaz, B., Tiffin, P., Young, N. D., and Sadowsky, M. J., 2013. Comparative genomics of the core and accessory genomes of 48 Sinorhizobium strains comprising five genospecies. Genome Biol. 14:R17.

Sugawara, M., and Sadowsky, M. J. 2014. Enhanced nodulation and nodule development by nolR mutants of Sinorhizobium medicae on specific Medicago host genotypes. Mol. Plant-Microbe Interact 27:328-335.

Suominen, L., Paulin, L., Saano, A., Saren, A. M., Tas, E., and Lindström, K. 1999. Identification of nodulation promoter (nod-box) regions of Rhizobium galegae. FEMS Microbiol. Lett. 177:217-223.

Tzfira, T., Vaidya, M., and Citovsky, V. 2004. Involvement of targeted proteolysis in plant genetic transformation by Agrobacterium. Nature 431:87-92.

Vergunst, A. C., Schrammeijer, B., den Dulk-Ras, A., de Vlaam, C. M. T., Regensburg-Tuïnk, T. J. G., and Hooykaas, P. J. J. 2000. VirB/D4dependent protein translocation from Agrobacterium into plant cells. Science 290:979-982.

Vergunst, A. C., van Lier, M. C., den Dulk-Ras, A., and Hooykaas, P. J. 2003. Recognition of the Agrobacterium tumefaciens VirE2 translocation signal by the VirB/D4 transport system does not require VirE1. Plant Physiol. 133:978-988.

Vergunst, A. C., van Lier, M. C. M., den Dulk-Ras, A., Stüve, T. A., Ouwehand, A., and Hooykaas, P. J. 2005. Positive charge is an important feature of the C-terminal transport signal of the VirB/D4-translocated proteins of Agrobacterium. Proc. Natl. Acad. Sci. U.S.A. 102:832-837.

Yang, S., Tang, F., Gao, M., Krishnan, H. B., and Zhu, H. 2010. $R$ genecontrolled host specificity in the legume-rhizobia symbiosis. Proc. Natl. Acad. Sci. U.S.A. 107:18735-18740.

Yasuda, M., Miwa, H., Masuda, S., Takebayashi, Y., Sakakibara, H., and Okazaki, S. 2016. Effector-triggered immunity determines host genotypespecific incompatibility in legume-Rhizobium Symbiosis. Plant Cell Physiol. 57:1791-1800.

\section{AUTHOR-RECOMMENDED INTERNET RESOURCES}

ApE, A Plasmid Editor: biologylabs.utah.edu/jorgensen/wayned/ape MaGe, the MicroScope Microbial Genome Annotation \& Analysis platform: https://www.genoscope.cns.fr/agc/microscope/home 